\title{
Coupling of Surface Acoustic Waves to a Two Dimensional Electron Gas
}

\author{
Steven H. Simon \\ Department of Physics, Massachusetts Institute of Technology, Cambridge, MA 02139.
}

\begin{abstract}
When a surface acoustic wave (SAW) is coupled piezoelectrically to a two dimensional electron gas (2DEG), a velocity shift and attenuation of the SAW are induced that reflect the conductivity of the 2DEG. This paper considers the case of a AlGaAs heterostructure with a 2DEG a distance $d$ from a (100) surface of the crystal where the SAWs are propagated in the [011] direction at wavevector $q$. It is found that the velocity shift $\Delta v_{s}$ and the attenuation coefficient $\kappa$ satisfy the well known equation $\left(\Delta v_{s} / v_{s}\right)-(i \kappa / q)=\left(\alpha^{2} / 2\right) /\left(1+\frac{i \sigma_{x x}(q, \omega)}{\sigma_{m}}\right)$ where $\sigma_{x x}(q, \omega)$ is the complex conductivity at wavevector $q$ and frequency $\omega=v_{s} q$ with $v_{s}$ the velocity of the SAW. The coefficients $\alpha$ and $\sigma_{m}$ are calculated and it is found that $\alpha$ has a nontrivial dependence on the product $q d$.
\end{abstract}

\section{INTRODUCTION}

For almost thirty years it has been known that the velocity $v_{s}$ of surface acoustic waves (SAWs) in piezoelectric crystals can beffffected by the electrical properties of nearby conductorst, 2 . If the nearby conductors are dissipative, then they can allow the SAW to attenuate also. Work by Ingebrigtsent, and later authors 3 showed that when a piezoelectric is brought next to a thin layer of a conducting medium, the $\mathrm{SAW}$ velocity shift $\Delta v_{s}$ and the attenuation coefficient $\kappa$ satisfy the relation

$$
\frac{\Delta v_{s}}{v_{s}}-\frac{i \kappa}{q}=\frac{\alpha^{2} / 2}{1+i \sigma_{x x}(q, \omega) / \sigma_{m}}
$$

where $\sigma_{x x}(q, \omega)$ is the longitudinal conductivity of the adjoining medium at wavevector $q$ and frequency $\omega=v_{s} q$. Note that the velocity shift is measured with respect to the velocity of the SAW when the adjoining medium has infinite conductivity. The coefficients $\sigma_{m}$ and $\alpha^{2} / 2$ depend on material parameters and are discussed at length in this paper.

Using the above relation between SAW velocity shift (or attenuation) and the conductivity of a surface layer, experimentalists have probed the conducting properties of two dimensional electron gases (2DEGs) placed near the surface of crystals of GaAst 10 .12. (An approximation of the experimental geometry is shown in Fig. 1.) In the earlier of these experiments 6 , the wavelength of the probing SAW was much larger than the distance $d$ from the surface. In this case, the depth $d$ can be neglected, and the coefficients $\sigma_{m}$ and $\alpha^{2} / 2$ can be assumed to he constant. However, in the more recent experiments 811 , the wavevector $q$ can be so large that the product $q d$ is or order unity. In this case, one must carefully consider the wavevector dependencies of these coefficients. Roughly one might expect that the coupling $\alpha^{2} / 2$ should decay approximately as $e^{-2 q d}$. However, it is seen experimentally that the coupling remains roughly constant up to the highest wavevectors probed $(q d \approx 4)$. In this paper, the wavevector dependences of $\sigma_{m}$ and $\alpha^{2} / 2$ are explicitly derived for an experimental geometry similar to that used in these experimental works. Using the results derived here, it should be possible to deduce quantitative results about the frequency and wavevector dependent conductivities of the samples (A detailed analysis of the data in References 9 and 10 is given in Reference 13.).

In References 511, the SAWs are propagated in the [011] direction along a (100) surface of an (Al)GaAs crystal. For most of this paper it will be sufficient to approximate this system as the geometry shown in Fig. 1. In other words we assume that the $2 \mathrm{DEG}$ is a thin conducting layer a distance $d$ (typically between 1000 and 5000 Angstroms) from the surface of a homogeneous $\mathrm{Al}_{x} \mathrm{Ga}_{1-x} \mathrm{As}$ crystal with the fraction $x$ of Al taken to be approximately $30 \%$. The effects of the differences between the actual experiments and this idealization will be considered in the concluding section of this paper.

The outline of the remainder of this paper is as follows. In section II, the electromagnetic response function $K_{00}$ is defined and related to the conductivity $\sigma_{x x}$. The parameter $\sigma_{m}$ is then defined in terms of the SAW velocity and the effective dielectric constant (ie the effective strength of the Coulomb interaction in the 2DEG). The effective dielectric constant is calculated explicitly in the appendix. In section III a qualitative explanation is given as to how the SAW induces a potential through piezoelectric coupling, thus resulting in an energy shift and/or dissipation through the real and/or imaginary part of the conductivity. Equation 1 is then derived leaving only the coupling constant $\alpha^{2} / 2$ to be calculated. In section IV the form of the SAW (neglecting the piezoelectric coupling) is discussed with particular focus on finding the energy density per unit area of the SAW. The effect of a small piezoelectric coupling is then considered in section Vyielding a form for the induced potential. The coupling $\alpha^{2} / 2$ is then derived and is found to have a nontrivial and nonmonotonic dependence on $q d$. Finally, section VI] considers a number of experimental issues and summarizes results. 


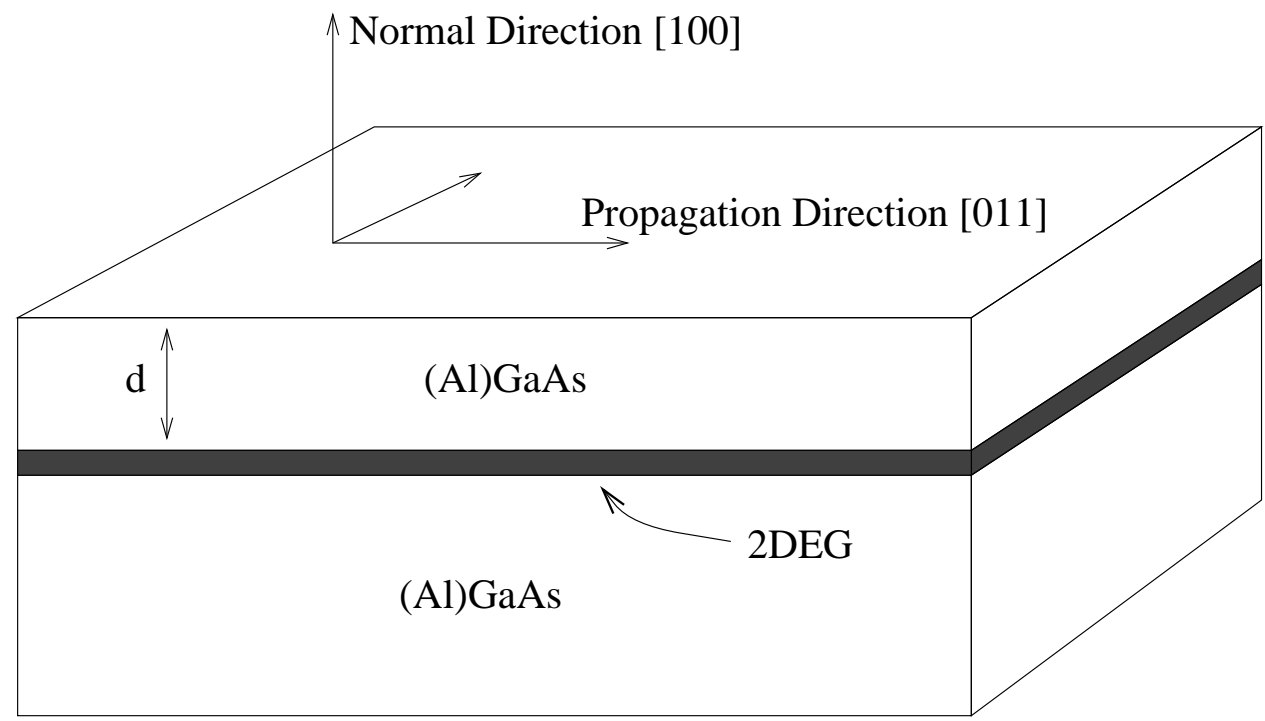

Fig. 1: Model Geometry for Surface Acoustic Wave Experiments. In the experiments the spacing $d$ is typically between 1000 and 5000 Angstroms. The $\mathrm{Al}_{x} \mathrm{Ga}_{1-x}$ As typically has a fraction of $\mathrm{Al}$ given by $x \approx 30 \%$.

\section{RESPONSE FUNCTIONS}

The density-density response $K_{00}(q, \omega)$ is defined by the relation

$$
n(q, \omega)=K_{00}(q, \omega) \phi^{\text {ext }}(q, \omega)
$$

where $\phi^{\text {ext }}$ is the perturbing externally applied scalar potential applied at a frequency $\omega$ and wavevector $\mathbf{q}=q \hat{\mathbf{x}}$, and $n(q, \omega)$ is the induced fluctuation density. As we will see below, the SAW experiments directly measure $K_{00}$ at finite frequency and wavevector.

Many linear response measurements do not measure, however, the ratio of induced density to the externally applied potential but rather the response to the total potential. A density $n(\mathbf{q})$ induced by the external vector potential, gives rise to a Coulomb scalar potential

$$
\phi^{\text {ind }}(q, \omega)=-v(q) n(q, \omega)
$$

where $v(q)=\frac{2 \pi}{\epsilon_{\text {eff }}}$ is the Fourier transform of the usual Coulomb interaction $v(r)=1 /\left(\epsilon_{\text {eff }}|r|\right)$. (In principle, currents in the sample give rise to an induced vector as well as scalar potential, but in practice these fields are negligible). Here $\epsilon_{\text {eff }}$ is the effective background dielectric constant. The wavevector dependent form of this dielectric constant for the case of the model geometry of Fig. 1 is derived in the appendix, and is given by

$$
\frac{\epsilon_{\text {eff }}}{\epsilon}=\frac{1}{2}\left(\frac{\left(\epsilon+\epsilon_{0}\right) \exp (q d)}{\epsilon \cosh (q d)+\epsilon_{0} \sinh (q d)}\right)
$$

where $\epsilon$ is the dielectric constant of the bulk $\mathrm{Al}_{x} \mathrm{Ga}_{1-x} \mathrm{As}$ and $\epsilon_{0}$ is the dielectric constant of the medium above the surface $(\approx 1)$. The dielectric constant 14 for $\mathrm{Al}_{x} \mathrm{Ga}_{1-x} \mathrm{As}$ with $x \approx .3$ is approximately 12.5 ( which is slightly lower than the dielectric constant for GaAs which is approximately 13.0).

Using Eq. 3, the total scalar potential

$$
\phi^{\text {tot }}=\phi^{\text {ext }}+\phi^{\text {ind }}
$$

is now written as

$$
\phi^{\mathrm{tot}}=\left(1-v(q) K_{00}\right) \phi^{\mathrm{ext}}
$$

It then becomes useful to define the polarization $\Pi_{00}(q, \omega)$, which relates the induced density $n(q, \omega)$ to the total scalar potential via

$$
n(q, \omega)=\Pi_{00}(q, \omega) \phi^{\text {tot }}(q, \omega)
$$

Combining this definition with Eqs. 2 and 6 yields the equation

$$
\left[K_{00}\right]^{-1}=\left[\Pi_{00}\right]^{-1}+v(q)
$$

Since the response function $\Pi_{00}$ relates the density to the total vector potential, it is useful to write this function in terms of the conductivity $\sigma_{\alpha \beta}$ which relates the two spatial components of the current $\left(j_{x}, j_{y}\right)$ to the two spatial components of the total electric field $\left(E_{x}, E_{y}\right)$ via $\mathbf{j}=\sigma \mathbf{E}$. Using current conservation to give $j_{x}=(\omega n / q)$, then yields

$$
\sigma_{x x}=\frac{-i \omega}{q^{2}} \Pi_{00}
$$

In particular, this allows us to write the general relation

$$
\left[K_{00}\right]^{-1}=v(q)-\frac{i \omega}{q^{2} \sigma_{x x}}
$$


Throughout this work, we will assume that $\omega=v_{s} q$ with $v_{s}$ the SAW velocity, which then implies

$$
K_{00}(q, \omega)=\frac{\epsilon_{\text {eff }} q}{2 \pi\left(1-i \sigma_{m} / \sigma_{x x}(q, \omega)\right)}
$$

with

$$
\sigma_{m}=\frac{v_{s} \epsilon_{\mathrm{eff}}}{2 \pi} .
$$

The function $\sigma_{m}(q d)$ is shown in Fig. 2. Here, the experimentally relevant parameters for References 9 and 10 are used. These are $\epsilon=12.5$ and $v_{s}=3010 \mathrm{~m} / \mathrm{sec}$ (see section IV below).

\section{INDUCED ENERGY SHIFT}

Due to the piezoelectric coupling, an external scalar potential $\phi^{\text {ext }}$ is induced in the 2DEG. For now, we will write

$$
\phi^{\mathrm{ext}}=C e_{14} F(q d) / \epsilon
$$

where $C$ is the amplitude of the SAW, $e_{14}$ the piezoelectric stress constant, and $F$ is a dimensionless function of $q d$ that represents the fact that the SAW decays into the bulk. Clearly $F$ should approach a constant as $q d \rightarrow 0$ and should approach zero as $q d \rightarrow \infty$. Roughly, one should expect that the function $F$ should decay as $e^{-q d}$.

The induced energy density per unit area due to this external potential is given by

$$
\delta U=\frac{1}{2} K_{00}\left|\phi^{\text {ext }}\right|^{2}
$$

This expression is obtained from integrating a differential $d \delta U=n\left(\phi^{\text {ext }}\right) d \phi^{\text {ext }}$ and using Eq. 2. (Note that using $\phi^{\text {tot }}$ here instead would account for only the electrical energy). Using Eq. 10 we can rewrite this shift as

$$
\delta U=\frac{\epsilon_{\text {eff }} q}{4 \pi\left(1-i \sigma_{m} / \sigma_{x x}(q, \omega)\right)}\left|\phi^{\text {ext }}\right|^{2}
$$

We now want to measure this energy shift with respect to the shift for $\sigma_{x x} \rightarrow \infty$. Thus,

$$
\begin{aligned}
\Delta U & \equiv \delta U-\delta U\left(\sigma_{x x}=\infty\right) \\
& =\frac{\epsilon_{\text {eff }} q}{4 \pi}\left[\frac{1}{1-i \sigma_{m} / \sigma_{x x}(q, \omega)}-1\right]\left|\phi^{\text {ext }}\right|^{2} \\
& =\frac{\epsilon_{\text {eff }} q}{4 \pi}\left[\frac{1}{1+i \sigma_{x x}(q, \omega) / \sigma_{m}}\right]\left|\phi^{\text {ext }}\right|^{2}
\end{aligned}
$$

It is found below that the surface acoustic wave has an energy density proportional to $C^{2} q^{2}$ where $C$ is the amplitude of the wave and $q$ is the wavevector. Furthermore, the wave decays exponentially into the bulk with a decay constant proportional to $q$. Thus, when integrated in the $\hat{\mathbf{z}}$ direction, the energy $U$ per unit surface area is given by

$$
U=q C^{2} H
$$

where $H$ is a factor that depends on material parameters that we will determine below. Combining this with the results of the above section, the fractional energy shift is then given by

$$
\frac{\Delta U}{U}=\frac{\alpha^{2} / 2}{1+i \sigma_{x x}(q, \omega) / \sigma_{m}}
$$

where

$$
\frac{\alpha^{2}}{2}=\frac{\epsilon_{\text {eff }}}{\epsilon} \frac{e_{14}^{2}}{4 \pi \epsilon H}|F(q d)|^{2}
$$

(Note that the factor of $4 \pi$ will vanish when $\epsilon_{\text {eff }}$ is converted into MKSI units). Clearly, this result implies the velocity shift and attenuation relation given by Eq. 1. All that now remains is the tedious job of evaluating the constant $H$ as well as the functional form $F$. It should be noted that in the small $q d$ limit, various experiments 1 , 50 have measured the value of the coupling constant and have found $\alpha^{2} / 2 \approx 3.2 \times 10^{-4}$. As is discussed below in section $\mathrm{VI}$, these measurements should be viewed with caution. As discussed above, one expects roughly that $F$ decays as $e^{-q d}$ so that $\alpha^{2} / 2$ decays as $e^{-2 q d}$. This, however, contradicts experimental observation 11 . Below, in a more careful analysis, we will see why the decay is actually somewhat slower and shows a nonmonotonic dependence on $q d$.

\section{NON-PIEZOELECTRIC SAWS}

We begin by discussing the solution of the SAW equations with the piezoelectric coupling set to zero. The piezoelectric coupling will then be added at lowest order.

Defining a displacement vector $u_{k}$, the elastic wave equation is given by 15 - 17

$$
c_{i j k l} \partial_{l} \partial_{i} u_{k}+\rho \ddot{u}_{j}=0
$$

where $\rho$ is the mass density, $c$ is the elastic tensor, we have used the notations $\partial_{l} f=\frac{\partial f}{\partial x_{l}},, \dot{f}=\frac{\partial f}{\partial t}$, and repeated indices are summed. For GaAs, AlAs, and other crystals of cubic symmetry there are only 3 independent elastic constants. These constants are conventionally called $c_{11}, c_{12}$, and $c_{44}$. For GaAs at low temperatures, the elastic constants $c_{11}, c_{12}$, and $c_{44}$ are given by $1212.26 \times 10^{10}, 5.71 \times 10^{10}$, and $9.90 \times 10^{10} \mathrm{~N} / \mathrm{m}^{2}$ respectively. The constants for AlAs 1920 are given approximately by $12.2 \times 10^{10}, 5.5 \times 10^{10}$, and $5.7 \times 10^{10} \mathrm{~N} / \mathrm{m}^{2}$ respectively. It is noted that the elastic constants of the two materials are roughly the same. For $\mathrm{Al}_{x} \mathrm{Ga}_{1-x} \mathrm{As}$ it is reasonable to interpolate for any value of $x$. (Experimeptally, there may be some uncertainty in $x$ ). The density 19 of GaAs is $5307 \mathrm{~kg} / \mathrm{m}^{3}$, and the density of AlAs is $3598 \mathrm{~kg} / \mathrm{m}^{3}$. Thus for $\mathrm{Al}_{x} \mathrm{Ga}_{1-x}$ As with $x \approx .3$, the 
density interpolates to approximately $4794 \mathrm{~kg} / \mathrm{m}^{3}$, which differs from that of GaAs by only $10 \%$.

In considering surface waves, the wave equation must be supplemented with the boundary condition at the free surface that there is no total force at the surface. This condition is written as 15.16

$$
c_{\hat{\mathbf{z}} j k l} \partial_{l} u_{k}=0 .
$$

where the subscript $\hat{\mathbf{z}}$ represents the direction normal to the surface. For certain geometries, analytic solutions of the SAW equations are available. In the present case of a (100) surface with wave propagation in the [011] direction, the velocity of SAW propagation is given by the solution (here we are interested in the lowest velocity solution) of the cubic equatione2n.

$$
\left(1-\frac{c_{11}}{c_{44}} X\right)\left(\frac{c_{11} c_{11}^{\prime}-c_{12}^{2}}{c_{11}^{2}}-X\right)^{2}=X^{2}\left(\frac{c_{11}^{\prime}}{c_{11}}-X\right)
$$

where $c_{11}^{\prime}=\frac{1}{2}\left(c_{11}+c_{12}+2 c_{44}\right)$ and $X=\rho v_{s}^{2} / c_{11}$ gives the SAW velocity $v_{s}$.

For $\mathrm{Al}_{x} \mathrm{Ga}_{1-x}$ As with $x \approx .3$, the velocity is approximately $3010 \mathrm{~m} / \mathrm{sec}$. (This differs from that of pure GaAs by only $5 \%$.) Once the velocity is determined, one can easily solve analytically for the form of the SAW. For the experimental geometry we are presently considering, the displacements for this wave can be written as 2122

$$
\begin{aligned}
u_{x} & =C\left(e^{-\Omega q z-i \varphi}+e^{-\Omega^{*} q z+i \varphi}\right) e^{i q\left(x-v_{s} t\right)} \\
i u_{z} & =C\left(\gamma e^{-\Omega q z-i \varphi}+\gamma^{*} e^{-\Omega^{*} q z+i \varphi}\right) e^{i q\left(x-v_{s} t\right)}
\end{aligned}
$$

with $u_{y}=0$ and $C$ the amplitude $(C$ has dimensions of length). Here, the $\hat{\mathbf{x}}$ direction is chosen in the direction of wave propagation (the [011] direction). The parameters $\Omega, \gamma$, and $\varphi$ are determined by 21.22

$$
\begin{aligned}
0 & =\left(c_{11}^{\prime}-X c_{11}-\Omega^{2} c_{11}\right)\left(c_{44}-X c_{11}-\Omega^{2} c_{44}\right) \\
\gamma & =\Omega\left[\frac{c_{12}^{2}+c_{44}\left(c_{12}+c_{44}\right)^{2}}{c_{44}-\left(X+\Omega^{2}\right) c_{11}}\right] \\
e^{-2 i \varphi} & =-\frac{\gamma^{*}-\Omega^{*}}{\gamma-\Omega}
\end{aligned}
$$

In the case of $\mathrm{Al}_{x} \mathrm{Ga}_{1-x} \mathrm{As}$ with $x=.3$, the values of $\gamma$ and $\Omega$ and $\varphi$ are given in this case by $\Omega \approx .501+.472 i$, $\gamma \approx-.705+1.146 i$, and $\phi \approx 1.06$.

The local energy density of this wave can be written as 15.17

$$
E=\frac{1}{2} c_{i j k l} u_{i j} u_{k l}^{*}
$$

where the strain $u_{i j}$ is given by

$$
u_{i j}=\frac{1}{2}\left(\partial_{i} u_{j}+\partial_{j} u_{i}\right)
$$

For the AlGaAs surface waye discussed above, the energy density can be written as 17

$$
\begin{aligned}
E=\frac{1}{2}\left(c_{11}^{\prime}\left|u_{x x}\right|^{2}+c_{11}\left|u_{z z}\right|^{2}\right. & \\
& \left.+2 c_{12} \operatorname{Re}\left[u_{x x}^{*} u_{z z}\right]+c_{44}\left|2 u_{x z}\right|^{2}\right)
\end{aligned}
$$

Inserting the above described form of the wave, yields the strains

$$
\begin{aligned}
u_{x x} & =i q u_{x} \\
u_{z z} & =i q C\left(\gamma \Omega e^{-\Omega q z-i \varphi}+\text { c.c. }\right) e^{i q\left(x-v_{s} t\right)} \\
2 u_{x z} & =C q\left([\gamma-\Omega] e^{-\Omega q z-i \varphi}+\text { c.c. }\right) e^{i q\left(x-v_{s} t\right)}
\end{aligned}
$$

with "c.c." meaning complex conjugate. Finally, integrating the result of Eq. 32 in the $\hat{\mathbf{z}}$ direction yields an energy per unit surface area in the form given by Eq. 19 with

$$
\begin{aligned}
H & =\operatorname{Re}\left[c_{11}^{\prime}\left(\frac{e^{-2 i \varphi}}{\Omega}+\frac{1}{\alpha}\right)\right. \\
& +c_{11}\left(\frac{(\gamma-\Omega)^{2} e^{-2 i \varphi}}{\Omega}+\frac{|\gamma-\Omega|^{2}}{\alpha}\right) \\
& +c_{44}\left(\left(\gamma^{2} \Omega\right) e^{-2 i \varphi}+\frac{|\gamma \Omega|^{2}}{\alpha}\right) \\
& \left.+2 c_{12}\left(\gamma e^{-2 i \varphi}+\frac{\operatorname{Re}(\gamma \Omega)}{\alpha}\right)\right]
\end{aligned}
$$

where $\alpha=\operatorname{Re}(\Omega)$. This yields a numerical value of

$$
H \approx 28.8 \times 10^{10} \mathrm{~N} / \mathrm{m}^{2}
$$

(The value for pure GaAs is lower by only about $2 \%$ ).

\section{PIEZOELECTRIC COUPLING}

When a piezoelectricfoupling is added, the wave equations take the form 1.6 .17

$$
\begin{gathered}
c_{i j k l} \partial_{l} \partial_{i} u_{k}+e_{k i j} \partial_{k} \partial_{i} \phi+\rho \ddot{u}_{j}=0 \\
e_{i k l} \partial_{l} \partial_{i} u_{k}-\epsilon \nabla^{2} \phi=0
\end{gathered}
$$

where $e$ is the piezoelectric stress tensor, $\phi$ is the electric potential, and $\epsilon$ is the dielectric constant of the medium (here $\epsilon$ is assumed to be isotropic). For GaAs, AlAs (and other cubic crystals of class $\overline{4} 3 \mathrm{~m}$ ), there is only one independent nonzero component of the piezoelectric tensor 17 called $e_{14}$.

The value of $e_{14}$ for GaAs has an accepted value 19 of approximately $.157 \mathrm{C} / \mathrm{m}^{2}$. However, it should be noted that there is a small amount of evidence 23 that the actual value might be somewhat lower (by perhaps as much as $40 \%$ ). For the present work we will choose to work with the accepted value. For the case of AlAs, it is even more 
difficult to find a reliable value for the piezoelectric coupling. To the author's knowledge, no reliable measurement of this quantity has been made to date24. Several calculations of $e_{14}$ have been made, and the results range from 25 as small as .02 $C / \mathrm{m}^{2}$ td 26 as large as .22 $\mathrm{C} / \mathrm{m}^{2}$. If we choose one of these for the value of $e_{14}$ for AlAs and interpolate to obtain $e_{14}$ for $\mathrm{Al}_{x} \mathrm{Ga}_{1-x} \mathrm{As}$ with $x={ }_{3}$ (linear interpolation is thought to be roughly correct 24 ) we will obtain results that range from approximately .11 $\mathrm{C} / \mathrm{m}^{2}$ to $.18 \mathrm{C} / \mathrm{m}^{2}$. Since since $e_{14}$ is squared in the final result (Eq. 21), these uncertainties will be magnified. Although this uncertainty results in an overall change in the magnitude of the coupling, it will not change the functional form of the coupling with respect to changes in $q d$. For definiteness, we will choose to work with a value of $e_{14}$ of $.145 \mathrm{C} / \mathrm{m}^{2}$ for $\mathrm{Al}_{x} \mathrm{Ga}_{1-x}$ As with $x \approx .3$, (which is close to the value for pure GaAs). The uncertainty is approximately $.04 \mathrm{C} / \mathrm{m}^{2}$. When this quantity is squared in Eq. 21, the final result has an uncertainty in scale of about $50 \%$.

Since the piezoelectric coupling $e_{14}$ is small, it is clear from the second equation that $\phi$ will be order $e_{14}$ smaller than $u$. Thus, the first equation will be solved by the nonpiezoelectric solution discussed above with corrections only at order $e_{14}^{2}$. The mechanical boundary conditions in the piezoelectric case ard 10

$$
c_{\hat{\mathbf{z}} j k l} \partial_{l} u_{k}+e_{k \hat{\mathbf{z}} j} \partial_{k} \phi=0 .
$$

Again, this will be satisfied by the nonpiezoelectric solution with corrections at order $e_{14}^{2}$. The electrical boundary condition that the normal component of electric displacement $\mathbf{D}$ is continuous across the surface can be written asl

$$
\begin{aligned}
\phi & =i \frac{v_{s}^{2}}{\omega} Z D_{\hat{\mathbf{z}}} \\
D_{\hat{\mathbf{z}}} & =e_{\hat{\mathbf{z}} k l} \partial_{l} u_{k}-\epsilon \partial_{k} \phi
\end{aligned}
$$

Where $Z$ is the transverse magnetic wave impedance ( $\left.Z=E_{x} / H_{y}\right)$ of the medium above the surface. The impedance of the adjoining medium can be written as

$$
Z=\frac{i}{v_{s} \epsilon_{0}}
$$

where $\epsilon_{0}$ is the adjoining medium's dielectric constant. These conditions can be rewritten as

$$
0=\left.\left(\epsilon_{0} q \phi+e_{\hat{\mathbf{z}} k l} \partial_{l} u_{k}-\epsilon \partial_{\hat{\mathbf{z}}} \phi\right)\right|_{z=0}
$$

Here, this boundary condition must be properly treated since it is of lower order in $e_{14}$. Thus, we will use the above nonpiezoelectric solution for $u$ and solve Eqs. 39 and 44 for $\phi$. These two equations in our case can be recast as

$$
\begin{aligned}
\epsilon \nabla^{2} \phi & =e_{14}\left(\partial_{z} u_{x x}+2 \partial_{x} u_{x z}\right) \\
0 & =\left.\left(\epsilon_{0} q \phi+e_{14} u_{x x}-\epsilon \partial_{\hat{\mathbf{z}}} \phi\right)\right|_{z=0}
\end{aligned}
$$

The proposed form of solution is

$$
\begin{aligned}
\phi=\frac{i C e_{14}}{\epsilon} e^{i q\left(x-v_{s} t\right)}\left[A_{1} e^{-\Omega q z-i \varphi}\right. & \\
& \left.+A_{2} e^{-\Omega^{*} q z+i \varphi}+A_{3} e^{-q z}\right]
\end{aligned}
$$

with $C$ the amplitude of the SAW. Eq. 45 immediately yields the conditions

$$
A_{1}=A_{2}^{*}=\frac{\gamma-2 \Omega}{\Omega^{2}-1}
$$

Finally, using Eq.46 yields

$$
A_{3}=\frac{-2}{1+r}\left[\cos \varphi+r \operatorname{Re}\left(A_{1} e^{-i \varphi}\right)+\operatorname{Re}\left(\Omega A_{1} e^{-i \varphi}\right)\right]
$$

with $r=\epsilon_{0} / \epsilon \approx \frac{1}{12.5}$
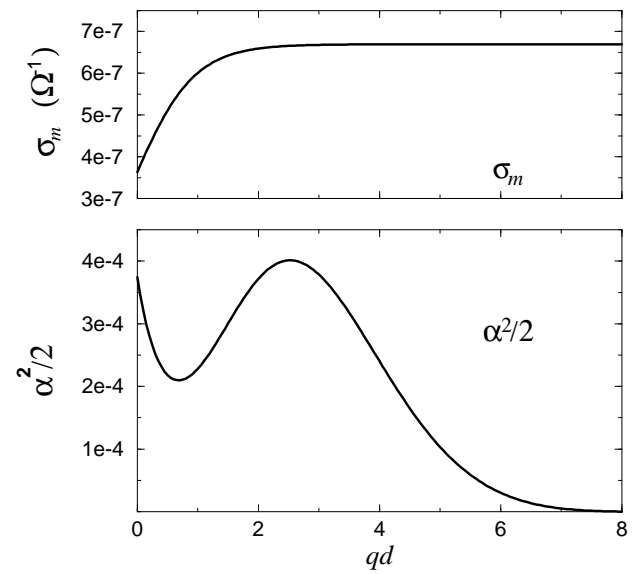

Fig. 2: Coupling Constants as a function of $q d$ with $q$ the wavevector and $d$ the distance from the 2DEG to the surface. Top: $\sigma_{m}(q d)$ in units of inverse Ohms. In this calculation, the dielectric constant of the medium $\left(\mathrm{Al}_{x} \mathrm{Ga}_{1-x} \mathrm{As}\right)$ is taken to be 12.5 . Bottom: $\alpha^{2}(q d) / 2$ (dimensionless). Here, the piezoelectric constant is taken as $.145 \mathrm{C} / \mathrm{m}^{2}$. The uncertainty in this number results in an uncertainty in the scale of approximately $50 \%$. Note that at $q d=0$ the coupling constant is roughly $3.7 \times 10^{-4}$ in good agreement with experiment.

Once the potential $\phi$ has been determined, this potential is then treated as the external potential $\phi^{\text {ext }}$ applied to the 2DEG. Note that the scalar potential $\phi^{\text {ind }}$ then induced by the density fluctuations in the 2DEG does not change the solution to Eqs 39 and 44 above since $\nabla^{2} \phi^{\text {ind }}=0$ everywhere outside of the $2 \mathrm{DEG}$ and $\epsilon_{0} q \phi^{\text {ind }}=\epsilon \partial_{\hat{\mathbf{z}}} \phi^{\text {ind }}$ at the surface (the solution of such a boundary condition is discussed in the appendix). The form of the potential is given by Eq. 13 where the functional dependence $F$ is given by

$$
F(q d)=2\left|A_{1}\right| e^{-\alpha q d} \cos (\beta q z+\phi+\xi)+A_{3} e^{-q d}
$$


where $\Omega=\alpha+\beta i$ and $A_{1}=\left|A_{1}\right| e^{-i \xi}$. Here we have the values $\left|A_{1}\right| \approx 1.59, \phi+\xi \approx 2.41, A_{3} \approx-3.10$. Using these values in Eq. 21 yields a the coupling constant $\alpha^{2} / 2$. The functional dependence of $\alpha^{2} / 2$ on $q d$ is shown in Fig. V. It is clear that the dependence is quite nontrivial. First of all, the exponential decay at large $q d$ is roughly proportional to $e^{-q d}$ rather than $e^{-2 q d}$. This is because, due to the precise material parameters, the SAW decays into the bulk as $e^{-\alpha q d}$ with $\alpha \approx \frac{1}{2}$. More importantly, at small $q d$ the coupling seems to oscillate. The reason for this is roughly that the boundary condition fixes the strain $u_{x z}$ to be zero at $z=0$. Thus $E_{x}(z=0)$ is mainly caused by the surface charge (ie, the $A_{3}$ term in Eq. 47). As $z$ (or $d$ ) increases, the effect of the surface charge term quickly decays, but the strain $u_{x z}$ becomes nonzero so that the coupling decays first, but then increases. Finally, at large $q d$, the exponential decay of the SAW damps out the coupling.

\section{CONCLUSION AND FURTHER CONSIDERATIONS}

This work has focused on surface acoustic waves in AlGaAs coupled to a 2DEG a distance $d$ away from the surface of the sample. The general relation (Eq. 1) between the fractional SAW velocity shift $\Delta v_{s} / v_{s}$, the attenuation $\kappa$ and the conductivity $\sigma_{x x}$ of the 2DEG was derived, and the coefficients $\alpha^{2} / 2$ and $\sigma_{m}$ were explicitly calculated as a function of the product $q d$ of the wavevector $q$ and the distance $d$ to the surface.

Although Eq. 11 is very general, the values of the coefficients $\sigma_{m}$ and $\alpha^{2} / 2$ are quite dependent on material parameters. As discussed in the text and in the appendix, $\sigma_{m}$ is dependent only on the velocity of the SAW and on the effective background dielectric constant in the 2DEG (which is in general wavevector dependent). The coupling constant $\alpha^{2} / 2$, on the other hand, is very sensitive to the details of the sample. In this paper we have focused only on a relatively simple model geometry where the sample is assumed to be a homogeneous slab of AlGaAs to simplify the solution of the wave problem. In actual experiments, the samples are often complicated many layer heterostructures. In the relevant experiments 11 , the bulk of the crystal (below the 2DEG) is pure GaAs and most of the crystal between the 2DEG and the surface is $\mathrm{Al}_{x} \mathrm{Ga}_{1-x}$ As with $x \approx .3$. However, additional thin layers of GaAs are added in this region, along with $\mathrm{Si}$ dopants. In principle, we could solve the wave equations for this complicated geometry and apply similar methods, but in practice such problems can only be solved numerically. However, since the elastic constants, densities, and dielectric constants of AlGaAs and GaAs are so similar we suspect that these heterostructures can be well approximated by the homogeneous system discussed here.

In using Eq. 11 to extract $\sigma_{x x}(q, \omega)$ from experimental data, there are several complications. To begin with, accurate measurements of the attenuation are extremely difficult, as are absolute measurements of the velocity 11 . However, measurements of the relative velocity shift can be made quite accurately. Another complication is that the above formula for the velocity shift (Eq. 11) gives the velocity shift $\Delta v_{s}$ relative to the velocity of the SAW if the conductivity of the $2 \mathrm{DEG}$ were infinite. In practice, the velocity shift is usually measured relative to the velocity of the SAW at zero magnetic field. It is often the case in high mobility samples (particularly at low frequency) that the conductivity at zero magnetic field is sufficiently large that it can be considered infinite and this approximation becomes reasonable. However, more generally, if the conductivity at zero field is well known, the resulting measured shift can be appropriately adjusted.

In References 510 , the parameters $\sigma_{m}$ and $\alpha^{2} / 2$ are both fit to experiment. To do this, the dc conductivity is measured and put into Eq. 1, the values of $\sigma_{m}$ and $\alpha^{2} / 2$ are then varied until a good fit is obtained to the experimentally measured values of $\Delta v_{s} / v_{s}$ as a function of magnetic field. There are several possible problems with this procedure. To begin with, the zero frequency (dc) conductivity is expected to be somewhat different from the finite frequency and wavevector conductivity $\sigma_{x x}\left(q, \omega=v_{s} q\right)$ that must be used in Eq. 1. Furthermore, there are indications that due to large scale inhomogeneities in the sample27, the measured dc conductivity may not accurately represent the spatial average of $\sigma_{x x}$. We thus conclude that these experimental fits of these parameters to the dc conductivity should be viewed with caution. Nonetheless the qualitative features of these experiments are relatively robust and many of the conclusions drawn from these experiments are relatively independent 8 of the precise value of the fit parameters $\sigma_{m}$ and $\alpha^{2} / 2$. A more careful quantitative analysis of these data is given in Reference 13 .

\section{ACKNOWLEDGMENTS}

It is a pleasure to acknowledge helpful discussions with Bertrand Halperin, Bob Willett, Eric Westerberg, and Konstantin Matveev. The author is also grateful to G. W. Farnell for sending computer code for numerically analyzing more complicated SAW problems. This work is supported by the National Science Foundation under grant numbers DMR-94-16910 and DMR-95-23361.

\section{APPENDIX A: COULOMB INTERACTION NEAR A DIELECTRIC INTERFACE}

The Coulomb interaction between electrons in the 2DEG is affected by the presence of the free surface of AlGaAs since the dielectric constant of the medium above the surface $\left(\epsilon_{0} \approx 1\right)$ is much less than the dielectric constant of AlGaAs $(\epsilon \approx 12.5)$. In this appendix, we consider the electrostatic problem of a charge in a 2DEG a distance $d$ from this AlGaAs/air interface. Consider a 
charge $e=1$ placed in the 2DEG at position $\mathbf{r}=0$ such that the AlGaAs surface is at the goordinate $z=d$. It is a standard result of electrostatics 28 that the electrostatic potential in the AlGaAs generated by such a charge is given by

$$
\Phi(\mathbf{r})=\frac{e}{\epsilon}\left(\frac{1}{|\mathbf{r}|}+\left[\frac{\epsilon-\epsilon_{0}}{\epsilon+\epsilon_{0}}\right] \frac{1}{|\mathbf{r}+2 \hat{\mathbf{z}} d|}\right) .
$$

Here, $|\mathbf{r}+2 \hat{\mathbf{z}} d|$ is the distance from $\mathbf{r}$ to the image charge, a distance $d$ away from the surface on the air side. Restricting $\mathbf{r}$ to lie in the plane of the 2DEG, and Fourier transforming, yields

$$
v(k)=\int d^{2} r e^{i k \cdot r} \Phi(\mathbf{r})
$$

which can be evaluated using Eqns. 6.564, 8.411, and 8.469.3 from Reference 29 to yield

$$
v(k)=\frac{2 \pi}{\epsilon_{\mathrm{eff}} k}
$$

where the effective dielectric constant is defined by

$$
\frac{1}{\epsilon_{\text {eff }}}=\frac{1}{\epsilon}\left(1+\left[\frac{\epsilon-\epsilon_{0}}{\epsilon+\epsilon_{0}}\right] e^{-2 k d}\right)
$$

which can be rewritten in the form of Eq. 4. Note that the effective dielectric constant ranges from $\epsilon$ for large $q d \gg 1$ to $\left(\epsilon+\epsilon_{0}\right) / 2$ for $q d \ll 1$.

${ }^{1}$ K. A. Ingebrigtsen, J. Appl. Phys. 40, 2681 (1969).

${ }^{2}$ C-C. Tseng and R. M. White, J. Appl. Phys. 38, 4274 (1967).

${ }^{3}$ P. Bierbaum, Appl. Phys. Lett. 21, 595 (1972).

${ }^{4}$ A. L. Efros, Y. M. Galperin, Phys. Rev. Lett. 64, 1959 (1990); Some of the calculations given in this work are very similar to those given here. However, in this reference, the 2DEG is assumed to be a distance $d$ above (ie, outside of) the piezoelectric.

${ }^{5}$ A. Wixforth, J. P. Kotthaus, and G. Weimann, Phys. Rev. Lett. 56, 2104 (1986)

${ }^{6}$ A. Wixforth et al, Phys Rev. B 40, 7874 (1989).

${ }^{7}$ R. L. Willett, M. A. Paalanen, R. R. Ruel, K. W. West, L. N. Pfieffer, and D. J. Bishop, Phys. Rev. Lett. 65, 112 (1990)

${ }^{8}$ R. L. Willett, R. R. Ruel, M. A. Paalanen, K. W. West, and L. N. Pfieffer, Phys. Rev. B 47, 7344 (1993)
${ }^{9}$ R. L. Willett, R. R. Ruel, K. W. West, and L. N. Pfieffer, Phys. Rev. Lett 71, 3846 (1993); see also R. L. Willett, Surf. Sci. 30576 (1994).

${ }^{10}$ R. L. Willett, K. W. West, and L. N. Pfieffer, Phys. Rev. Lett 75, 2988 (1995).

${ }^{11}$ R. L. Willett, personal communication.

${ }^{12}$ For related work, see A. Schenstrom et al, Solid State Comm. 65, 739 (1988); V. W. Rampton et al, Semicond. Sci. Tech. 7, 641 (1992); A. Esslinger et al, Surf. Sci. 305 83 (1994); J. M. Shilton et al, J. Phys. Cond. Mat. 77675 (1995).

13 S. H. Simon, B. I. Halperin, and R. L. Willett, to be published.

${ }^{14}$ Handbook of Optical Constants of Solids ed. E. D. Palik (Academic, Boston, 1985); Handbook of Optical Constants of Solids II ed. E. D. Palik (Academic, Boston, 1991).

${ }^{15}$ L. D. Landau and E. M. Lifshitz, Theory of Elasticity, 3ed, (Pergamon, UK, 1986).

${ }^{16}$ G. W. Farnell in Physical Acoustics v. 6 ed. W. P. Mason and R. N. Thurston (Academic Press, London, 1970); See also G. W. Farnell in Acoustic Surface Waves ed. A. A. Oliner (Springer-Verlag, NY, 1978).

${ }^{17}$ B. A. Auld Acoustic Fields and Waves in Solids v. 1 (Wiley, NY, 1973).

${ }^{18}$ See also T. W. Grudowski and M. Gilden, Appl. Phys. Lett. 38, 412 (1980). Noted that the experimental geometry used in this reference is slightly different from that considered here.

${ }^{19}$ M. Neuberger, Handbook of Electronic Materials v.2. (Plenum, NY, 1971); and references therein.

${ }^{20}$ N. Chetty, A. Muñoz, and R. M. Martin, Phys. Rev. B.40, 11934 (1989); and references therein.

${ }^{21}$ R. Stonely, Proc. Royal. Soc. A232 447 (1955).

${ }^{22}$ It should be noted that in Eq. 25 the forms of the solutions assume that the two roots of Eq. 28 are complex conjugate pairs $\left(\Omega\right.$ and $\left.\Omega^{*}\right)$. This is indeed the case for AlGaAs systems. However, for systems with somewhat smaller anisotropy (ie, when $c_{11}$ closer to $c_{11}^{\prime}$ ) the two roots can become real and unequal and the form of solution shown here is not correct.

23 J. L. Sanchez-Rojas et. al, Appl. Phys. Lett. 65, 2042 (1994); and references therein.

${ }^{24}$ S. Adachi, Physical Properties of III-V Semiconductor Compounds, (Wiley, NY, 1992); and references therein.

${ }^{25}$ S. D. Gironcoli et. al, Phys. Rev. Lett. 62, 2853 (1989).

${ }^{26}$ K. Hubner, Phys. Status. Solidi B24, 627 (1973).

27 S. H. Simon and B. I. Halperin, Phys. Rev. Lett. 73, 3278 (1994); I. Ruzin, N. R. Cooper, and B. I. Halperin, Phys. Rev. B 531558 (1996); I. Ruzin, N. R. Cooper, S. H. Simon, and B. I. Halperin, to be published.

28 J. D. Jackson, Classical Electrodynamics, 2ed, (Wiley, NY, 1975) p. 147.

${ }^{29}$ I. S. Gradshteyn and I. M. Ryzhik, Table of Integrals, Series, and Products, (Academic Press, San Diego, 1980). 